\title{
Methicillin-Resistant Staphylococcus aureus Nasal Carriage Among Patients and Healthcare Workers in a Hospital in Kelantan, Malaysia
}

\author{
HASSANAIN AL-TALIB ${ }^{1 *}$, CHAN YEAN YEAN ${ }^{2}$, HABSAH HASAN² ${ }^{2}$ NIK ZURAINA NMN ${ }^{2}$ \\ and MANICKAM RAVICHANDRAN ${ }^{3}$
}

${ }^{1}$ Faculty of Medicine, Universiti Teknologi MARA, Sungai Buloh, Malaysia

${ }^{2}$ Department of Medical Microbiology and Parasitology, School of Medical Sciences Universiti Sains Malaysia, Kota Bharu, Malaysia

${ }^{3}$ Faculty of Applied Sciences, AIMST University, Kedah, Malaysia

Received 19 April 2012, revised 26 September 2012, accepted 17 December 2012

\section{Abstract}

Staphylococcus aureus nasal carriage is a common source of nosocomial infection and colonization. The aim of the present study was to assess the burden of methicillin-resistant $S$. aureus nasal carriage, its association with factors of interest including its genetic relationships. The prevalence of S. aureus nasal carriage was found to be $28.7 \%$. This study showed that patients with a history of previous antibiotic intake, nasogastric tube, and longer hospitalization had a significantly high risk of being MRSA nasal carriers. The genetic relationship of all 34 nasal MRSA isolates revealed four major clusters of isolates, and there was a relationship between MRSA isolated from inpatients and healthcare workers.

Key words: MRSA, nasal carriage, nosocomial infection

Staphylococcus aureus is a common cause of hospital and community-acquired infections worldwide. S. aureus colonization is mainly found in the anterior nares (40\%) (Kaplan, 2005). Several worldwide studies have reported that the rate of nasal carriage of $S$. aureus varies from $16.8 \%$ to $90 \%$ (Alghaithy et al., 2000; Goyal et al., 2002). In a study conducted in several hospitals in Malaysia in 1996, the prevalence rate was $40 \%$ (Rohani et al., 2000). The principal mode of transmission from patient to patient is via the transiently colonized hands of healthcare workers (HCWs), who acquire the organism from patient contact or by handling contaminated materials (Solberg, 2000). Nasal carriage of S. aureus is a potential source of infection and colonization often precedes infection. In general, nasal carriage rates among hospital personal and patients (60-70\%) are much higher compared to those among community carriers (30-50\%) (Lowy, 1998). Identification and isolation of persons who are colonized or infected with methicillin-resistant S. aureus (MRSA) is recommended to minimize the spread of MRSA within hospitals. During 2005-2007, we identified an ongoing increase of both MRSA and S. aureus infection rates in Hospital Universiti Sains Malaysia (HUSM) despite high compliance with intensified control measures that were implemented during admission (Al-Talib et al., 2010). Thus, this study was carried out to determine the prevalence of $S$. aureus nasal carriage, and MRSA burden at HUSM, Kelantan state, Malaysia. This study was carried out at HUSM which is a 747-bed, tertiary care, teaching hospital. It is the referral center for Kelantan state and the nearby states, and is located at Kubang Kerian, which is about $7 \mathrm{~km}$ from Kota Bharu. The Research and Ethics Committee, School of Medical Sciences, Universiti Sains Malaysia, approved the study protocol. This was a cross-sectional study that was carried out from 1 March to 31 August 2008 among patients, relatives and staff nurses in all of the wards in HUSM. A total of 550 subjects were included in this study. Informed consent was obtained from all participants before specimen collection. Epidemiological information was obtained by interviewing the participants at the time that the nasal swabs were collected. Demographic (sex, race, and occupation) and clinical conditions data (history of antibiotic usage in the past 2 weeks, duration of hospitalization, and history of fever in the past 2 weeks) were included in this study. The specimens were taken from the subjects by using a sterile moistened swab inserted into the nostril, to a depth of approximately $1 \mathrm{~cm}$, and rotated five times.

* Corresponding author: H. Al-Talib, Faculty of Medicine, Universiti Teknologi MARA, Sungai Buloh, Malaysia; phone: +60 355442839 ; fax: +60 355442831 ; e-mail: hassanainiy@yahoo.com 
For each subject, both nostrils were sampled using the same swab. Trypticase soy broth was used as the transport medium. The specimens were quickly sent to the laboratory and inoculated onto mannitol salt agar (MSA) plates. All colonies surrounded by yellow zones on MSA after $48 \mathrm{~h}$ of incubation at $37^{\circ} \mathrm{C}$ were selected. Colonies with pink or red zones were excluded. Identification of S. aureus was made based on the colony characteristics on MSA, Gram staining and a positive tube coagulase test. Molecular typing was conducted using pulsed-field gel electrophoresis (PFGE) as described by McDougal et al. (McDougal et al., 2003). Restriction digestion was performed with SmaI, and restriction fragments were separated in a CHEF-DRII system (Bio-Rad, Hercules, CA, USA). Analysis of DNA fragments was conducted using both visual inspection and Bio-Rad software. Strain relatedness was interpreted according to published guidelines (Tenover et al., 1995). A total of 550 Staphylococcus species were isolated from the anterior nares of patients, relatives and HCWs. The total prevalence of $S$. aureus nasal carriage was $28.7 \%$, which was higher than that in other studies (Choi et al., 2006; Santhosh, 2008). Norazah et al., in a study conducted in Malaysia in 2002, reported a higher prevalence of $S$. aureus nasal carriage which varied from $45 \%$ to $76 \%$ (Norazah et al., 2001). By analyzing all subjects with $S$.aureus nasal colonization, 34 were found to be colonized with MRSA and 124 were methicillin-sensitive S. aureus (MSSA) nasal carriers. S. aureus and particularly MRSA pose a major hazard in hospitals and contribute to the wider problem of hospital-acquired infection (Aldeyab et al., 2008). A marked increase in the prevalence of MRSA infections has been reported in Malaysia since the mid-1985 (Choi et al., 2006). A wide range of MRSA nasal carriage rates have been observed (Choi et al., 2006; Askarian et al., 2009; Johnson et al., 2009), which could be attributed to different study populations, interpretation guidelines and culture techniques. Out of these 550 Staphylococcus species isolates, 312 were isolated from inpatients, 156 were from patients accompanied relatives, and 82 were from HCWs. Among the 550 nasal swabs, 392 (71.3\%) were found to be coagulase-negative staphylococci and 158 (28.7\%) were S. aureus. Among the 158 S. aureus isolates, $34(21.5 \%)$ were found to be MRSA.

Surgical wards and ICUs showed higher percentages of MRSA nasal carriage (35.3\% and 29\%, respectively). This could be due to the traumatic and postoperative immunological suppression of the patients. The higher percentage with MRSA isolates from the surgical wards and ICUs in our study could be due to environmental factors that were probably related to the higher rate of MRSA infection in these locations. The higher proportion of MRSA nasal carriers among Malay race was due to the large proportion of that ethnic group in the
Table I

Socio-demographic characteristics of 158 MRSA/MSSA nasal carriers

\begin{tabular}{|c|c|c|c|}
\hline \multirow[b]{2}{*}{ Variables } & $\operatorname{MRSA}(n=34)$ & $\operatorname{MSSA}(n=124)$ & \multirow[b]{2}{*}{$P$ value } \\
\hline & $\begin{array}{c}\text { No. of subjects } \\
(\%)\end{array}$ & $\begin{array}{c}\text { No. of subjects } \\
(\%)\end{array}$ & \\
\hline $\begin{array}{l}\text { Sex } \\
\text { Male }\end{array}$ & $24(70.6)$ & $52(41.9)$ & \multirow[t]{2}{*}{$0.036^{\star *}$} \\
\hline Female & $10(29.4)$ & $72(58.1)$ & \\
\hline $\begin{array}{l}\text { Race } \\
\text { Malay }\end{array}$ & $30(88.2)$ & $118(95.2)$ & \multirow[t]{2}{*}{$0.292^{*}$} \\
\hline Chinese & $4(11.8)$ & $6(4.8)$ & \\
\hline $\begin{array}{l}\text { Age group (yr) } \\
<18\end{array}$ & $6(17.6)$ & $6(4.8)$ & \\
\hline $18-30$ & $8(23.5)$ & $30(24.2)$ & \\
\hline $31-45$ & $2(5.9)$ & $52(41.9)$ & \\
\hline $45-60$ & $14(41.2)$ & $26(21.0)$ & \\
\hline$>61$ & $4(11.8)$ & $10(8.1)$ & \\
\hline $\begin{array}{l}\text { Level of education } \\
\text { Tertiary }\end{array}$ & $4(11.8)$ & $12(9.7)$ & \\
\hline Secondary & $16(47)$ & $56(45.2)$ & \\
\hline Primary & $2(5.9)$ & $10(8.1)$ & \\
\hline Not educated & $12(35.3)$ & $46(37)$ & \\
\hline
\end{tabular}

${ }^{*}$ Fisher's exact test; ${ }^{* *}$ Pearson $\chi^{2}$ test

present study (Table I). There are many risk factors for MRSA nasal colonization including age, sex, duration of hospitalization and previous antibiotic treatment (Corea et al., 2003). In our multivariate analysis, the only significant independent risk factors for nasal carriage of MRSA versus MSSA were patients with a history of previous antibiotic intake, longer duration of hospitalization, and nasogastric intubation $(P=0.005$, $0.006,0.013$, respectively). Previous studies on factors associated with outbreaks of infection and MRSA nasal colonization have reported similar findings (Corea et al., 2003; Marshall et al., 2003). Routine screening of all patients for MRSA on admission is impractical due to financial and other constraints. Screening high-risk groups for MRSA carriage and instituting control measures might limit introduction of new strains into the hospital. In our study, patients with a history of antibiotic treatment had a 9.7-fold greater chance of being nasal carriers of MRSA than MSSA. Previous antibiotic administration has been shown to be significantly associated with acquisition of MRSA as compared to MSSA. Oztoprak et al. have reported that $86 \%$ of patients with previous antibiotic treatment developed MRSA infection compared to $63 \%$ who developed MSSA infection (Oztoprak et al., 2006). Schaefer et al. have shown that $41 \%$ of MRSA nasal carriers had received two or more antibiotics compared to $21 \%$ in the control group (Schaefer et al., 2009). The excessive use and abuse of antibiotics are the main contributing factors to bacte- 
Table II

Multiple logistic regression for the associated factors of MRSA nasal colonization.

\begin{tabular}{|l|c|c|c|c|c|c|}
\hline \multirow{2}{*}{ Variables } & \multirow{2}{*}{ B } & \multirow{2}{*}{ Wald } & \multirow{2}{*}{ Adjusted OR* } & \multicolumn{2}{c|}{ (95\%CI) } & \multirow{2}{*}{$P$ value } \\
\cline { 5 - 8 } & & & & Lower & Upper & \\
\hline Previous antibiotic intake & 2.275 & 0.035 & 9.72 & 2.017 & 45.97 & 0.005 \\
\hline Duration of hospitalization & 0.74 & 7.433 & 1.077 & 1.02 & 1.136 & 0.006 \\
\hline Nasogastric tube & 1.96 & 6.2 & 7.15 & 1.155 & 32.121 & 0.013 \\
\hline
\end{tabular}

rial resistance; therefore, antibiotics must be prescribed only when indicated, and the drug chosen is the most narrow spectrum agent, and should be given with appropriate dose and duration. Nasogastric intubation represents one of the main risk factors for nosocomial acquisition of MRSA (Erbay et al., 2003), and our study showed that it was significantly $(P=0.013)$ associated with MRSA nasal carriage, which could have been due to interference of the nasogastric tube with the normal lining epithelium of the upper respiratory tract, or the tube acting as a vehicle that facilitated transmission of MRSA to the respiratory tract (Table II). Another important confounder was the duration of hospital stay. The excess length of hospital stay was significantly $(P=0.006)$ longer among MRSA carriers than MSSA carriers. Therefore, an increase of 1 day duration of hospitalization increases by 1.077 times the acquisition risk of MRSA nasal colonization. Our findings were comparable to those of two previous studies. In Belgium, Blot et al. have reported that the mean length of stay for MRSA patients was 29 days as compared to 10 days for those with MSSA (Blot et al., 2002). Another study has found that length of stay $>16$ days was significantly associated with MRSA nosocomial infection (Wang et al., 2008). These studies have indicated that patients who require longer hospitalization usually have more severe underlying illness or are critically ill.
Therefore, we consider that longer hospitalization is a risk factor for transmission of MRSA among patients. Many hospitals have recently implemented active surveillance to identify MRSA nasal carriers among patients, accompanying relatives, and HCWs, however, ongoing MRSA transmission can persist in endemic environments despite active surveillance (Ben-David et al., 2008). There are several possible explanations for ongoing nosocomial MRSA transmission, including poor compliance with obtaining surveillance cultures, unscreened patients who are not included among highrisk patients, poor hand hygiene compliance, transmission of MRSA during the $72 \mathrm{~h}$ before colonized patients are identified and isolated, and insufficient measures to clean the contaminated environment. The genetic relationship of all 34 MRSA isolates was determined by PFGE (Fig. 1). A dendrogram of similarity percentage was calculated using dice coefficients from the PFGE data, based on an $80 \%$ cutoff value, which revealed four major clusters of isolates that were designated as pulsed-field type A-D. In the present study, four of the HCWs from surgical and ICUs wards were found to be MRSA nasal carriers. PFGE fingerprinting showed that the dominant PFGE pattern $\mathrm{C}$ was the dominant strain in 28 subjects $(82.35 \%)$, and found in HCWs and patients. However, patterns A and B were found in another four patients, and pattern $\mathrm{D}$ in two patients'

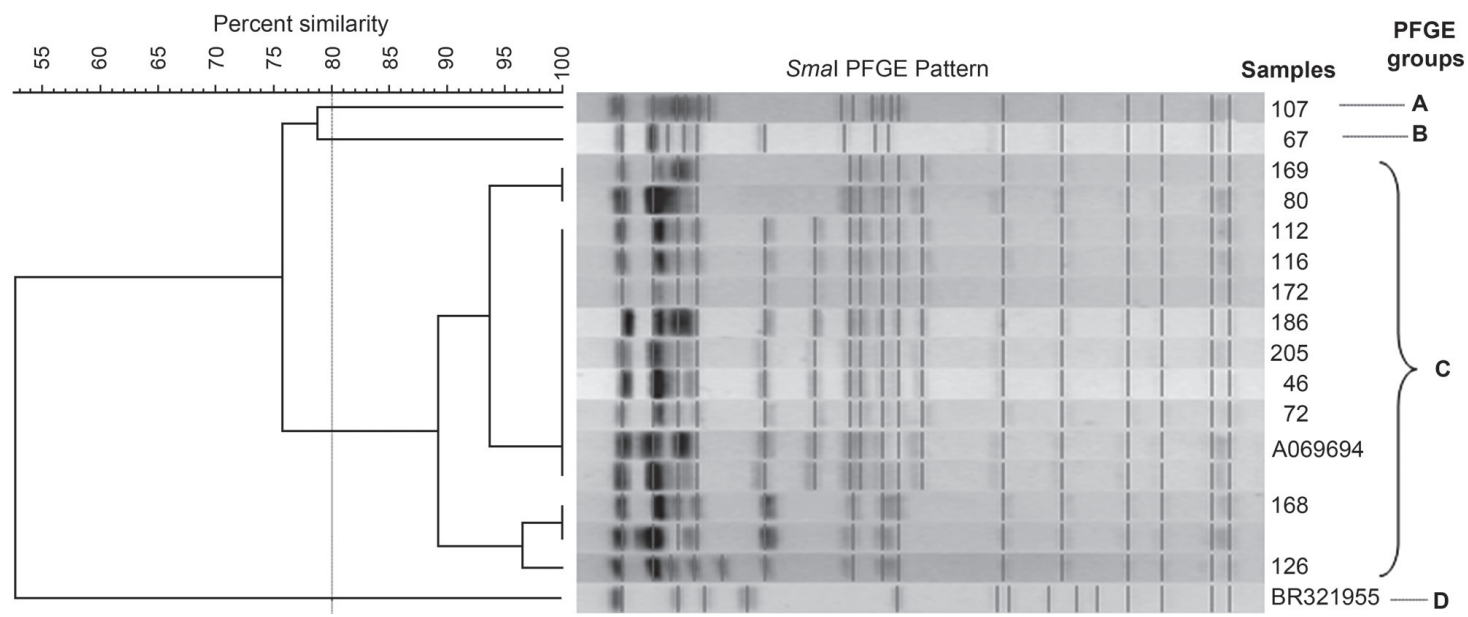

Fig. 1. Dendrogram of nasal MRSA samples

$(107,67,169,80,112,116,172,186,205,46,72$, A069694, Staff 2 intan, 168, staffICU, 126, BR321955) and PFGE groups A, B, C, and D 
relatives. There was a relationship between MRSA isolated from inpatients and HCWs. Unfortunately, our evidence is still insufficient to prove spread of MRSA between inpatients and HCWs, hence, more epidemiological information about MRSA isolates from HCWs and health residences would be useful. More surveys should be focused on HCWs' behavior because directcontact droplets and air-borne transmission seem to be the major routes of transmission in nosocomial infection (http://www.cdc.gov/mmwr/PDF/rr/rr5116.pdf.). Therefore, hand washing, and use of gloves, masks, eye protection, and face shields should be advocated as standard precautions for HCWs (Popovic et al., 2000). In conclusion, this study revealed that the prevalence of nasal carriage of S. aureus strains among the HUSM population was higher than that found in other studies in our country. Logistic regression showed that previous antibiotic intake, longer hospitalization and nasogastric intubation were independently associated with MRSA carriage. PFGE fingerprinting of the MRSA isolated from patients was related to that of HCWs.

\section{Acknowledgements}

This study was supported by Short-term grant (304/PPSP/ 6131535) from Universiti Sains Malaysia. We are grateful to Institute for postgraduate studies, Universiti Sains Malaysia for their Fellowship support, and Department of Medical Microbiology and Parasitology, Hospital Universiti Sains Malaysia, Kelantan, Malaysia.

\section{Literature}

Al-Talib H.I., C.Y. Yean, K. Al-Jashamy and H. Hasan. 2010. Methicillin-resistant Staphylococcus aureus nosocomial infection trends in Hospital Universiti Sains Malaysia during 2002-2007. Ann. Saudi. Med. 30: 358-363.

Aldeyab M.A., D.L. Monnet, J.M. Lopez-Lozano, C.M. Hughes, M.G. Scott, M.P. Kearney, F.A. Magee and J.C. McElnay. 2008. Modelling the impact of antibiotic use and infection control practices on the incidence of hospital-acquired methicillin-resistant Staphylococcus aureus: a time-series analysis. J. Antimicrob. Chemoth. 62: 593-600

Alghaithy A.A., N.E. Bilal, M. Gedebou and A.H. Weily. 2000 Nasal carriage and antibiotic resistance of Staphylococcus aureus isolates from hospital and non-hospital personnel in Abha, Saudi Arabia. T. Roy. Soc. Trop. Med. H. 94: 504-507.

Askarian M., A. Zeinalzadeh, A. Japoni, A. Alborzi and Z.A. Memish. 2009. Prevalence of nasal carriage of methicillin-resistant Staphylococcus aureus and its antibiotic susceptibility pattern in healthcare workers at Namazi Hospital, Shiraz, Iran. Int. J. Infect. Dis. 13: e241-247.

Ben-David D., L.A. Mermel and S. Parenteau. 2008. Methicillinresistant Staphylococcus aureus transmission: the possible importance of unrecognized health care worker carriage. Am. J. Infect. Control. 36: 93-97.

Blot S.I., K.H. Vandewoude, E.A. Hoste and F.A. Colardyn. 2002. Outcome and attributable mortality in critically Ill patients with bacteremia involving methicillin-susceptible and methicillin-resistant Staphylococcus aureus. Arch. Intern. Med. 162: 2229-2235.

Choi C.S., C.S. Yin, A.A. Bakar, Z. Sakewi, N.N. Naing, F. Jamal and N. Othman. 2006. Nasal carriage of Staphylococcus aureus among healthy adults. J. Microbiol. Immunol. Infect. 39: 458-464.
Clinical Laboratory Standards Institute in Molecular Diagnostic Methods for Infectious Diseases; Approved Guideline (CLSI MM3-A2) n.

Corea E., T. de Silva and J. Perera. 2003. Methicillin-resistant Staphylococcus aureus: prevalence, incidence and risk factors associated with colonization in Sri Lanka. J. Hosp. Infect. 55: 145-148. Erbay H., A.N. Yalcin, S. Serin, H. Turgut, E. Tomatir, B. Cetin and M. Zencir. 2003. Nosocomial infections in intensive care unit in a Turkish university hospital: a 2-year survey. Intens. Care. Med. 29: $1482-1488$.

Goyal R., S. Das and M. Mathur. 2002. Colonisation of methicillin resistant Staphylococcus aureus among health care workers in a tertiary care hospital of Delhi. Ind. J. Med. Sci. 56: 321-324. http://www.cdc.gov/mmwr/PDF/rr/rr5116.pdf.

Johnson L.B., J. Jose, F. Yousif, J. Pawlak and L.D. Saravolatz. 2009. Prevalence of colonization with community-associated methicillin-resistant Staphylococcus aureus among end-stage renal disease patients and healthcare workers. Infect. Cont. Hosp. Ep. 30: 4-8.

Kaplan S.L. 2005. Implications of methicillin-resistant Staphylococcus aureus as a community-acquired pathogen in pediatric patients. Infect. Dis. Clin. Am. 19: 747-757.

Lowy F.D. 1998. Staphylococcus aureus infections. N. Eng. J. Med. 339: 520-532.

Marshall C., G. Harrington, R. Wolfe, C.K. Fairley, S. Wesselingh and D. Spelman. 2003. Acquisition of methicillin-resistant Staphylococcus aureus in a large intensive care unit. Infect. Cont. Hosp. Ep. 24: 322-326.

McDougal L.K., C.D. Steward, G.E. Killgore, J.M. Chaitram, S.K. McAllister and F.C. Tenover. 2003. Pulsed-field gel electrophoresis typing of oxacillin-resistant Staphylococcus aureus isolates from the United States: establishing a national database. J. Clin. Microbiol. 41: 5113-5120.

Norazah A., Y.T. Koh, A. Ghani Kamel, R. Alias and V.K. Lim. 2001. Mupirocin resistance among Malaysian isolates of methicillinresistant Staphylococcus aureus. Int. J. Antimicrob. Ag. 17: 411-414. Oztoprak N., M.A. Cevik, E. Akinci, M. Korkmaz, A. Erbay, S.S. Eren, N. Balaban and H. Bodur. 2006. Risk factors for ICUacquired methicillin-resistant Staphylococcus aureus infections. Am. J. Infect. Control. 34: 1-5.

Popovic T., I.K. Mazurova, A. Efstratiou, J. Vuopio-Varkila, M.W. Reeves, A. De Zoysa, T. Glushkevich and P. Grimont. 2000. Molecular epidemiology of diphtheria. J. Infect. Dis. 181 Suppl 1: S168-177.

Rohani M.Y., A. Raudzah, M.G. Lau, A.A. Zaidatul, M.N. Salbiah K.C. Keah, A. Noraini and T. Zainuldin. 2000. Susceptibility pattern of Staphylococcus aureus isolated in Malaysian hospitals. Int. J.Antimicrob. Ag. 13: 209-213.

Santhosh DV S.K., I. Bairy, R. Gowrish and J. D’Souza. 2008 Phenotypic Detection and Rate of Nasal Carriage of Heterotypic Borderline Oxacillin Resistant Staphylococcus aureus in Pre-clinical Medical Students from Malaysia. J. Clin. Diag. Res. 2: 985-990.

Schaefer A.M., K.M. McMullen, J.L. Mayfield, A. Richmond, D.K. Warren and E.R. Dubberke. 2009. Risk factors associated with methicillin-resistant Staphylococcus aureus colonization on hospital admission among oncology patients. Am. J. Infect. Control. 37: 603-605.

Solberg C.O. 2000. Spread of Staphylococcus aureus in hospitals: causes and prevention. Scand. J. Infect. Dis. 32: 587-595.

Tenover F.C., R.D. Arbeit, R.V. Goering, P.A. Mickelsen, B.E. Murray, D.H. Persing and B. Swaminathan. 1995. Interpreting chromosomal DNA restriction patterns produced by pulsed-field gel electrophoresis: criteria for bacterial strain typing. J. Clin. Microbiol. 33: 2233-2239.

Wang F.D., Y.Y. Chen, T.L. Chen and C.Y. Liu. 2008. Risk factors and mortality in patients with nosocomial Staphylococcus aureus bacteremia. Am. J. Infect. Control. 36: 118-122. 